\title{
Analysis of an Inventory System for Items with Price-Dependent Demand and Time Dependent Three-Parameter Weibull Deterioration Function
}

\author{
0. Nwoba Pius', I. E. Chukwu Walford², S. Maliki Olaniyi ${ }^{3}$ \\ ${ }^{1}$ Department of Industrial Mathematics and Applied Statistics, Ebonyi State University, Abakaliki, Nigeria \\ ${ }^{2}$ Department of Statistics, University of Nigeria, Nsuka, Nigeria \\ ${ }^{3}$ Department of Mathematics, Michael Okpara University of Agriculture, Umudike, Nigeria \\ Email: ophokenshiphd@gmail.com,walford.chukwu@unn.edu.ng, somaliki@gmail.com
}

How to cite this paper: Pius, O.N., Chukwu Walford, I.E. and Maliki Olaniyi, S. (2021) Analysis of an Inventory System for Items with Price-Dependent Demand and Time Dependent Three-Parameter Weibull Deterioration Function. American Journal of Operations Research, 11, 199-214.

https://doi.org/10.4236/ajor.2021.114012

Received: April 19, 2021

Accepted: July 27, 2021

Published: July 30, 2021

Copyright $\odot 2021$ by author(s) and Scientific Research Publishing Inc. This work is licensed under the Creative Commons Attribution International License (CC BY 4.0).

http://creativecommons.org/licenses/by/4.0/

(c) (i) Open Access

\begin{abstract}
In this research work we propose a mathematical model of an inventory system with time dependent three-parameter Weibull deterioration and pricedependent demand rate. The model incorporates shortages and deteriorating items are considered in which inventory is depleted not only by demand but also by decay, such as, direct spoilage as in fruits, vegetables and food products, or deterioration as in obsolete electronic components. Furthermore, the rate of deterioration is taken to be time-proportional, and a power law form of the price dependence of demand is considered. This price-dependence of the demand function is nonlinear, and is such that when price of a commodity increases, demand decreases and when price of a commodity decreases, demand increases. The objective of the model is to minimize the total inventory costs. From the numerical example presented to illustrate the solution procedure of the model, we obtain meaningful results. We then proceed to perform sensitivity analysis of our model. The sensitivity analysis illustrates the extent to which the optimal solution of the model is affected by slight changes or errors in its input parameter values.
\end{abstract}

\section{Keywords}

Inventory, EOQ Model, Deterioration, Shortages, Weibull Distribution, Price-Dependent Demand, Sensitivity Analysis, MathCAD14

\section{Introduction}

The classical inventory model such as presented by Harris-Wilson [1] considers 
the ideal situation in which depletion of inventory is caused by a constant demand rate alone. However, it was observed afterwards that depletion of inventory may take place due to deterioration. Virtually all items deteriorate over time with the exception of items such as hardware, glassware, steel etc. On the other hand, all perishables such as food items, chemicals etc. deteriorate quite rapidly over time and become unsuitable for consumption. This change or deterioration can be defined as any process that prevents an item from being used for its intended original purpose. Following its utility, the deteriorating item can be characterized into either an item whose functionality or physical fitness deteriorates over time (e.g. fresh food or medicine) or an item whose functionality does not degrade, but where demand deteriorates over time as customers' perceived utility decreases. Both categories pertain to the same problem but require different actions seeing that items that lose their functional characteristics and quality often cannot, or should not be kept in inventory. However, items that lose perceived utility can be kept in inventory and may be sold on a secondary market. This loss must be taken into account when analyzing inventory systems. In recent times several research articles on the above subject have appeared in the literature including Chakrabarti [2], Covert and Philip [3] developed a two-parameter Weibull distribution deterioration for an inventory model. This investigation was followed by Datta and Pal [4], Jalan et al. [5], Dixit and Shah [6], Giri et al. [7], Shah et al. [8] and more recently Nwoba et al. [9] analyzed an inventory system for items with stochastic demand and time dependent three-parameter Weibull deterioration function.

The main objective of inventory management for deteriorating items is to obtain optimal returns during the useful lifetime of the product [10]. This leads to three main issues: determining reasonable and appropriate methods for issuing inventory, replenishing inventory and allocating inventory. The choice of inventory valuation methods adopted in issuing inventory (i.e. the order in which the items are to be issued), such as methods based on time sequence including FIFO (first-in, first-out) and LIFO (last-in, first-out), depends on both the intrinsic characteristics of the inventory (e.g. lifetime, quantity, variety, issuing frequency etc.) and the influence on the company (e.g. inventory balance, cost of goods sold etc.) [11]. In the present paper, we consider an Economic Order Quantity (EOQ) model for inventory of items that deteriorate following a time dependent three-parameter Weibull deterioration and price-dependent demand rate.

\subsection{Mathematical Formulation}

A rich literature on modelling of deteriorating inventory shows how the deterioration of products has been captured in the research problem up till now. To integrate deterioration into mathematical models, the model type (deterministic or stochastic) and the considered time horizon (infinite or finite) lead to specific methods [12]. In what follows we consider the basic characteristics of EOQ inventory models. 


\subsection{The Demand Characteristics}

The customer arrival rate per time period may be deterministic or stochastic, each individual demand may be deterministic or stochastic and each individual demand may also be discrete or continuous [13] [14]. Demand plays a key role in the modeling of deteriorating inventory. Aiming towards satisfying customer demand, companies employ demand forecasts as a prediction of customer behaviour. The following variations of demand labeled from the point of view of real life situations have been recognized and studied by a number of researchers such as Khanra et al. [15]. It is assumed that demand is known with certainty in a deterministic demand process. Stochastic demand process on the other hand basically incorporates randomness and unpredictability.

A deterministic demand distribution can be categorized into:

1) Uniform demand, i.e. demand is a constant, fixed number of items.

2) Time-varying demand.

3) Stock-dependent demand.

4) Price-dependent demand.

A combination of the above is also possible.

\subsection{Price-Dependent Demand Function}

It is observed that, the demand rate of an item is influenced by the selling price of an item, as, whenever the selling price of an item increases, the demand decreases and vice-versa. Generally, this type of demand is seen for finished goods. Several authors have investigated this type of inventory model. According to the market research, it is observed that time to time advertisement of an item can also affect its demand. The demand rates of these items may be dependent on displayed stock level. Such types of demand in different forms were considered by Maiti [16], Chung et al. [17]. All these models considered either linear or non-linear form of demand and derived results. In this research work we propose a demand rate that is a non-linear function of time $t$, and given by

$$
D(t)=d(p) \omega \eta[t-(t-\mu) H(t-\mu)]^{\eta-1} ; 0<\omega<1, \eta>0,
$$

where

$$
H(t-\mu)= \begin{cases}1, & t \geq \mu \\ 0, & t<\mu\end{cases}
$$

is the well-known Heaviside function, with graph shown in Figure 1.

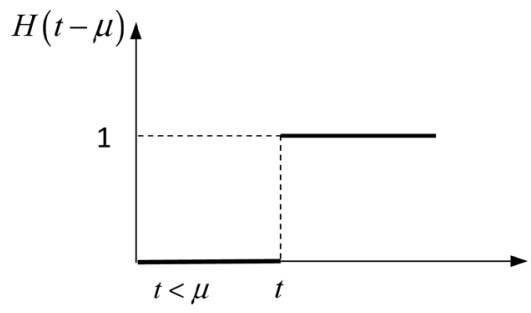

Figure 1. The Heaviside function. 
The functional form of $d(p)$ for this research is prescribed as $d(p)=a p^{-b}$, where $a>0$ is a scale parameter and $b>0$ is a shape parameter.

\subsection{Proposed Deterioration Model}

The Weibull distribution is specified by;

$$
W(t)=\alpha \beta(t-\gamma)^{\beta-1} \exp \left(-\alpha(t-\gamma)^{\beta}\right), t>0,
$$

is often used for modeling lifetime data. When modeling monotone hazard rates, the Weibull distribution may be an initial choice because of its negatively and positively skewed density shape. Rinne [18] suggested that a three-parameter generalization of the Weibull distribution deals with general situations in modeling survival process with various shapes in the hazard function. Chakrabarty et al. provided rationale for considering three-parameter Weibull deterioration rate. They discovered that many products that start deteriorating appreciably only after a certain period (e.g. after they are produced) and for which the rate of deterioration increases over time have a deterioration rate best described by a Weibull distribution. We consider the following inventory data adapted from Ghosh and Chaudhuri [19] and Saha and Chakrabarti [20].

\subsection{Notations of the Model}

We adopt the following notations and assumptions in the derivation of our model.

Notations:

1) $I(t)$ : On-hand inventory as a function of time $t$.

2) $\theta(t)=\alpha \beta(t-\gamma)^{\beta-1}$ : instantaneous deterioration rate function for a three-parameter Weibull distribution given in (2); where $\alpha$ is the scale parameter, $\beta$ is the shape parameter and $\gamma$ is the location parameter $(0<\alpha \ll 1)$.

3) $D(t):$ Demand function of time $t$.

4) $P(t)$ : Production rate as a function of time $t$.

5) T: is the fixed length of each production cycle (cycle time).

6) $c_{1}:$ is the inventory holding cost per unit per unit time.

7) $c_{2}:$ is the shortage cost per unit per unit time.

8) $c_{3}:$ is the cost of each deteriorated unit.

9) $\phi$ : total average cost.

10) $I_{0}$ : is initial inventory size.

11) $t_{1}$ : time during which there is no shortage.

12) $\kappa:$ a constant value between 0 and 1 .

13) $T^{*}$ : optimal value of $T$.

14) $p^{*}$ : optimal value of $p$.

15) $\phi^{*}$ : optimal value of $\phi$.

16) $I_{0}^{*}$ : optimal value of $I_{0}$.

17) $t_{1}^{*}$ : optimal value of $t_{1}$. 


\subsection{Assumptions of the Model}

1) The inventory system under consideration deals with single item.

2) Replenishment size is constant.

3) Lead time is zero.

4) The planning horizon is infinite.

5) The demand rate is non-linear as a function of time $t$, and specified by Equation (1).

6) Shortages in the inventory are allowed and completely backlogged.

7) The supply is instantaneous and the lead time is zero.

8) Deteriorated unit is not repaired or replaced during a given cycle.

9) The holding cost, ordering cost, shortage cost and unit cost remain constant over time.

10) There are no quantity discounts.

11) The distribution of the time to deterioration of the items follows the three-parameter Weibull distribution.

\section{The Mathematical Model}

At the beginning of the cycle, the inventory level $I(t)$ reaches its maximum $I(0)=I_{0}$ units of item at time $t=0$. During the interval $\left[0, t_{1}\right]$, the inventory level depletes due to the combine effects of demand and deterioration. At $t=t_{1}$, the inventory level is zero and all the demand hereafter (i.e. $T-t_{1}$ ) is completely backlogged. The total number of backordered items is replaced by the next replenishment. A graphical representation of this inventory system is depicted in Figure 2. Since the depletion of the units is due to demand and deterioration, the rate of change of the inventory level at any time $t$ is governed by the differential equations:

$$
\frac{\mathrm{d} I(t)}{\mathrm{d} t}+\theta(t) I(t)=P(t)-D(t), \quad 0 \leq t<t_{1}
$$

with boundary conditions $I(0)=I_{0}$ and $I\left(t_{1}\right)=0$. Furthermore the production rate $P(t)$ is zero in this case, thus in the interval $0 \leq t<\mu$, the initial value problem to be solved is:

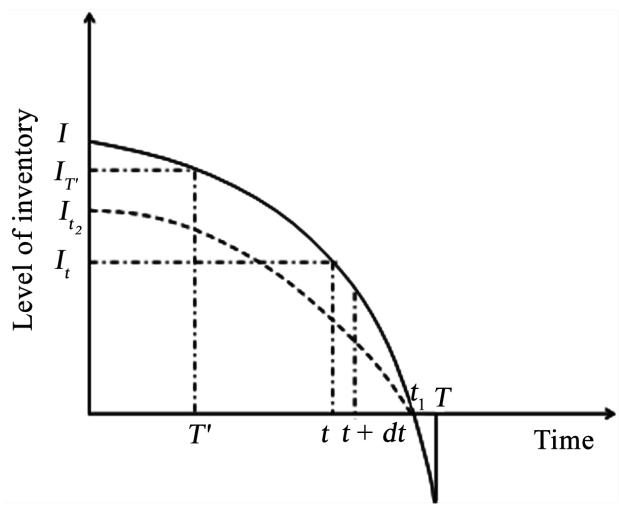

Figure 2. An EOQ model with shortages and deterioration. 


$$
\frac{\mathrm{d} I(t)}{\mathrm{d} t}+\theta(t) I(t)=-D(t), \quad I(0)=I_{0}, I\left(t_{1}\right)=0
$$

In the interval $t_{1} \leq t \leq T$, the initial value problem becomes:

$$
\frac{\mathrm{d} I(t)}{\mathrm{d} t}=-d(p) \omega \eta t^{\eta-1}, \quad I\left(t_{1}\right)=0
$$

Employing the previously stated assumptions, we have our inventory model as:

$$
\begin{gathered}
\frac{\mathrm{d} I(t)}{\mathrm{d} t}+\alpha \beta(t-\gamma)^{\beta-1} I(t)=-d(p) \omega \eta t^{\eta-1}, \quad 0 \leq t<\mu \\
\frac{\mathrm{d} I(t)}{\mathrm{d} t}+\alpha \beta(t-\gamma)^{\beta-1} I(t)=-d(p) \omega \eta \mu^{\eta-1}, \quad \mu \leq t<t_{1} \\
\frac{\mathrm{d} I(t)}{\mathrm{d} t}=-d(p) \omega \eta t^{\eta-1}, \quad t_{1} \leq t \leq T
\end{gathered}
$$

\subsection{Solution of the Model}

Equation (6) is a first order differential equation and its integrating factor is:

$$
\exp \left[\alpha \beta \int(t-\gamma)^{\beta-1} \mathrm{~d} t\right]=\mathrm{e}^{\alpha(t-\gamma)^{\beta}}
$$

So its solution is given as

$$
\begin{gathered}
\frac{\mathrm{d}}{\mathrm{d} t}\left[I(t) \mathrm{e}^{\alpha(t-\gamma)^{\beta}}\right]=-d(p) \omega \eta t^{\eta-1} \mathrm{e}^{\alpha(t-\gamma)^{\beta}} \\
\therefore \quad\left[I(t) \mathrm{e}^{\alpha(t-\gamma)^{\beta}}\right]_{0}^{t}=-d(p) \omega \eta \int_{0}^{t} s^{\eta-1} \mathrm{e}^{\alpha(s-\gamma)^{\beta}} \mathrm{d} s \\
\Rightarrow I(t) \mathrm{e}^{\alpha(t-\gamma)^{\beta}}=I_{0} \mathrm{e}^{\alpha(-\gamma)^{\beta}}-d(p) \omega \eta \int_{0}^{t} s^{\eta-1} \mathrm{e}^{\alpha(s-\gamma)^{\beta}} \mathrm{d} s
\end{gathered}
$$

i.e.

$$
I(t)=I_{0} \mathrm{e}^{\alpha(-\gamma)^{\beta}-\alpha(t-\gamma)^{\beta}}-d(p) \omega \eta \mathrm{e}^{-\alpha(t-\gamma)^{\beta}} \int_{0}^{t} s^{\eta-1} \mathrm{e}^{\alpha(s-\gamma)^{\beta}} \mathrm{d} s, \quad 0 \leq t<\mu .
$$

where $I_{0}=I(0)$. For Equation (7) we follow the same procedure

$$
\begin{gathered}
\frac{\mathrm{d}}{\mathrm{d} t}\left[I(t) \mathrm{e}^{\alpha(t-\gamma)^{\beta}}\right]=-d(p) \omega \eta \mu^{\eta-1} \mathrm{e}^{\alpha(t-\gamma)^{\beta}} \\
\therefore \quad\left[\mathrm{e}^{\alpha(t-\gamma)^{\beta}} I(t)\right]_{\mu}^{t}=-d(p) \omega \eta \mu^{\eta-1} \int_{\mu}^{t} \mathrm{e}^{\alpha(s-\gamma)^{\beta}} \mathrm{d} s \\
\Rightarrow \quad \mathrm{e}^{\alpha(t-\gamma)^{\beta}} I(t)=\mathrm{e}^{\alpha(\mu-\gamma)^{\beta}} I(\mu)-d(p) \omega \eta \mu^{\eta-1} \int_{\mu}^{t} \mathrm{e}^{\alpha(s-\gamma)^{\beta}} \mathrm{d} s
\end{gathered}
$$

i.e.

$$
I(t)=I_{\mu} \mathrm{e}^{\alpha\left[(\mu-\gamma)^{\beta}-(t-\gamma)^{\beta}\right]}-d(p) \omega \eta \mu^{\eta-1} \mathrm{e}^{-\alpha(t-\gamma)^{\beta}} \int_{\mu}^{t} \mathrm{e}^{\alpha(s-\gamma)^{\beta}} \mathrm{d} s, \quad \mu \leq t<t_{1}
$$

where $I_{\mu}=I(\mu)$. On integrating Equation (8) we get;

$$
I(t)=-d(p) \omega\left(t^{\eta}-t_{1}^{\eta}\right), \quad t_{1} \leq t \leq T
$$

Since $I\left(t_{1}\right)=0$, from Equation (10) we get; 


$$
\begin{gathered}
I\left(t_{1}\right)=I_{0} \mathrm{e}^{\alpha(-\gamma)^{\beta}-\alpha\left(t_{1}-\gamma\right)^{\beta}}-d(p) \omega \eta \mu^{\eta-1} \mathrm{e}^{-\alpha\left(t_{1}-\gamma\right)^{\beta}} \int_{0}^{t_{1}} \mathrm{e}^{\alpha(s-\gamma)^{\beta}} \mathrm{d} s=0 \\
\therefore I_{0} \mathrm{e}^{\alpha(-\gamma)^{\beta}-\alpha\left(t_{1}-\gamma\right)^{\beta}}=d(p) \omega \eta \mu^{\eta-1} \mathrm{e}^{-\alpha\left(t_{1}-\gamma\right)^{\beta}} \int_{0}^{t_{1}} \mathrm{e}^{\alpha(s-\gamma)^{\beta}} \mathrm{d} s \\
\Rightarrow I_{0}=d(p) \omega \eta \mu^{\eta-1} \mathrm{e}^{-\alpha(-\gamma)^{\beta}} \int_{0}^{t_{1}} \mathrm{e}^{\alpha(s-\gamma)^{\beta}} \mathrm{d} s
\end{gathered}
$$

Similarly, we can obtain $I_{\mu}$ using $I\left(t_{1}\right)=0$. Hence

$$
\begin{gathered}
I\left(t_{1}\right)=\mathrm{e}^{\alpha\left[(\mu-\gamma)^{\beta}-\left(t_{1}-\gamma\right)^{\beta}\right]} I_{\mu}-d(p) \omega \eta \mu^{\eta-1} \mathrm{e}^{-\alpha\left(t_{1}-\gamma\right)^{\beta}} \int_{\mu}^{t_{1}} \mathrm{e}^{\alpha(s-\gamma)^{\beta}} \mathrm{d} s=0 \\
\Rightarrow \quad I_{\mu}=d(p) \omega \eta \mu^{\eta-1} \mathrm{e}^{-\alpha(\mu-\gamma)^{\beta}} \int_{\mu}^{t_{1}} \mathrm{e}^{\alpha(s-\gamma)^{\beta}} \mathrm{d} s
\end{gathered}
$$

Hence, the inventory level at any time $t \in[0, T]$ is given by

$$
I(t)= \begin{cases}I_{0} \mathrm{e}^{\alpha(-\gamma)^{\beta}-\alpha(t-\gamma)^{\beta}}-d(p) \omega \eta \mathrm{e}^{-\alpha(t-\gamma)^{\beta}} \int_{0}^{t} s^{\eta-1} \mathrm{e}^{\alpha(s-\gamma)^{\beta}} \mathrm{d} s, & 0 \leq t<\mu \\ I_{\mu} \mathrm{e}^{\alpha\left[(\mu-\gamma)^{\beta}-(t-\gamma)^{\beta}\right]}-d(p) \omega \eta \mu^{\eta-1} \mathrm{e}^{-\alpha(t-\gamma)^{\beta}} \int_{\mu}^{t} \mathrm{e}^{\alpha(s-\gamma)^{\beta}} \mathrm{d} s, & \mu \leq t<t_{1} \\ -d(p) \omega\left(t^{\eta}-t_{1}^{\eta}\right), & t_{1} \leq t \leq T\end{cases}
$$

The total quantity of deteriorated items in the time interval $\left[0, t_{1}\right]$ is given by

$$
\begin{gathered}
D=\text { Initial inventory }- \text { Total demand within }\left[0, t_{1}\right] \\
=I_{0}-\left\{\text { Total demand within }[0, \mu]+\text { Total demand within }\left[\mu, t_{1}\right]\right\} \\
=I_{0}-\int_{0}^{\mu} D(t) \mathrm{d} t-\int_{\mu}^{t_{1}} D(t) \mathrm{d} t \\
=I_{0}-\left[\int_{0}^{\mu} d(p) \omega \eta t^{\eta-1} \mathrm{~d} t+\int_{\mu}^{t_{1}} d(p) \omega \eta \mu^{\eta-1} \mathrm{~d} t\right] \\
\quad \therefore \quad D=I_{0}+d(p) \omega t_{1}^{\eta}+d(p) \omega \eta \mu^{\eta-1}\left(t_{1}-\mu\right)
\end{gathered}
$$

Or

$$
D=d(p) \omega\left[t_{1}^{\eta}+\eta \mu^{\eta-1}\left(t_{1}-\mu\right)+\eta \mu^{\eta-1} \mathrm{e}^{-\alpha(-\gamma)^{\beta}} \int_{0}^{t_{1}} \mathrm{e}^{\alpha(s-\gamma)^{\beta}} \mathrm{d} s\right]
$$

The average total cost per unit time is given by

$$
\phi\left(T, t_{1}, p\right)=\frac{C_{3} D}{T}+\frac{C_{1}}{T} \int_{0}^{t_{1}} I(t) \mathrm{d} t-\frac{C_{2}}{T} \int_{t_{1}}^{T} I(t) \mathrm{d} t
$$

We observe that the integral $\int_{0}^{t_{1}} I(t) \mathrm{d} t$ in Equation (18) can be broken up into

$$
\int_{0}^{t_{1}} I(t) \mathrm{d} t=\int_{0}^{\mu} I(t) \mathrm{d} t+\int_{\mu}^{t_{1}} I(t) \mathrm{d} t
$$

Now

$$
\begin{aligned}
\int_{0}^{\mu} I(t) \mathrm{d} t & =\int_{0}^{\mu}\left\{I_{0} \mathrm{e}^{\alpha(-\gamma)^{\beta}-\alpha(t-\gamma)^{\beta}}-d(p) \omega \eta \mathrm{e}^{-\alpha(t-\gamma)^{\beta}} \int_{0}^{t} s^{\eta-1} \mathrm{e}^{\alpha(s-\gamma)^{\beta}} \mathrm{d} s\right\} \mathrm{d} t \\
& =I_{0} \mathrm{e}^{\alpha(-\gamma)^{\beta}} \int_{0}^{\mu} \mathrm{e}^{-\alpha(t-\gamma)^{\beta}} \mathrm{d} t-d(p) \omega \eta \int_{0}^{\mu} \mathrm{e}^{-\alpha(t-\gamma)^{\beta}} \mathrm{d} t \int_{0}^{t} s^{\eta-1} \mathrm{e}^{\alpha(s-\gamma)^{\beta}} \mathrm{d} s
\end{aligned}
$$

and

$$
\begin{aligned}
\int_{\mu}^{t_{1}} I(t) \mathrm{d} t & =\int_{\mu}^{t_{1}}\left\{I_{\mu} \mathrm{e}^{\alpha\left[(\mu-\gamma)^{\beta}-(t-\gamma)^{\beta}\right]}-d(p) \omega \eta \mu^{\eta-1} \mathrm{e}^{-\alpha(t-\gamma)^{\beta}} \int_{\mu}^{t} \mathrm{e}^{\alpha(s-\gamma)^{\beta}} \mathrm{d} s\right\} \mathrm{d} t \\
& =I_{\mu} \int_{\mu}^{t_{1}} \mathrm{e}^{\alpha\left[(\mu-\gamma)^{\beta}-(t-\gamma)^{\beta}\right]} \mathrm{d} t-d(p) \omega \eta \mu^{\eta-1} \int_{\mu}^{t_{1}} \mathrm{e}^{-\alpha(t-\gamma)^{\beta}} \mathrm{d} t \int_{0}^{t} \mathrm{e}^{\alpha(s-\gamma)^{\beta}} \mathrm{d} s
\end{aligned}
$$


The last integral in (19) is computed as:

$$
\begin{aligned}
\int_{t_{1}}^{T} I(t) \mathrm{d} t & =-d(p) \omega \int_{t_{1}}^{T}\left(t^{\eta}-t_{1}^{\eta}\right) \mathrm{d} t \\
& =-d(p) \omega\left[\left(\frac{T^{\eta+1}}{\eta+1}-t_{1}^{\eta} T\right)-\left(\frac{t_{1}^{\eta+1}}{\eta+1}-t_{1}^{\eta+1}\right)\right]
\end{aligned}
$$

Hence, we have:

$$
\begin{aligned}
& \phi\left(T, t_{1}, p\right)=\frac{c_{3} \omega d(p)}{T}\left(t_{1}^{\eta}+\eta \mu^{\eta-1}\left(t_{1}-\mu\right)+\eta \mu^{\eta-1} \mathrm{e}^{-\alpha(-\gamma)^{\beta}} \int_{0}^{t_{1}} \mathrm{e}^{\alpha(s-\gamma)^{\beta}} \mathrm{d} s\right) \\
& +\frac{c_{1}}{T}\left\{I_{0} \mathrm{e}^{\alpha(-\gamma)^{\beta}} \int_{0}^{\mu} \mathrm{e}^{-\alpha(t-\gamma)^{\beta}} \mathrm{d} t-d(p) \omega \eta \int_{0}^{\mu} \mathrm{e}^{-\alpha(t-\gamma)^{\beta}} \mathrm{d} t \int_{0}^{t} s^{\eta-1} \mathrm{e}^{\alpha(s-\gamma)^{\beta}} \mathrm{d} s\right\} \\
& +\frac{c_{1}}{T}\left\{I_{\mu} \mathrm{e}^{\alpha(\mu-\gamma)^{\beta}} \int_{\mu}^{t_{1}} \mathrm{e}^{-\alpha(t-\gamma)^{\beta}} \mathrm{d} t-d(p) \omega \eta \mu^{\eta-1} \int_{\mu}^{t_{1}} \mathrm{e}^{-\alpha(t-\gamma)^{\beta}} \mathrm{d} t \int_{0}^{t} \mathrm{e}^{\alpha(s-\gamma)^{\beta}} \mathrm{d} s\right\} \\
& +\frac{c_{2} \omega d(p)}{T}\left[\left(\frac{T^{\eta+1}}{\eta+1}-t_{1}^{\eta} T\right)-\left(\frac{t_{1}^{\eta+1}}{\eta+1}-t_{1}^{\eta+1}\right)\right]
\end{aligned}
$$

Our objective now is to determine the values of $T, t_{1}$ and $p$ which minimize $\phi\left(T, t_{1}, p\right)$. However, at this stage we must prescribe the functional form of $d(p)$ which is given by $d(p)=a p^{-b}, a, b>0$.

We assume $t_{1}=\kappa T ; 0<\kappa<1$. This assumption appears reasonable since the length of the shortage interval is a fraction of the cycle time. Substituting $t_{1}=\kappa T$ in Equation (21), we get:

$$
\begin{aligned}
& \phi(T, p)=\frac{c_{3} \omega a p^{-b}}{T}\left(\kappa^{\eta} T^{\eta}+\eta \mu^{\eta-1}(\kappa T-\mu)+\eta \mu^{\eta-1} \mathrm{e}^{-\alpha(-\gamma)^{\beta}} \int_{0}^{\kappa T} \mathrm{e}^{\alpha(s-\gamma)^{\beta}} \mathrm{d} s\right) \\
& +\frac{c_{1}}{T}\left\{I_{0} \mathrm{e}^{\alpha(-\gamma)^{\beta}} \int_{0}^{\mu} \mathrm{e}^{-\alpha(t-\gamma)^{\beta}} \mathrm{d} t-a p^{-b} \omega \eta\left(\int_{0}^{\mu} \mathrm{e}^{-\alpha(t-\gamma)^{\beta}} \mathrm{d} t\right)\left(\int_{0}^{t} s^{\eta-1} \mathrm{e}^{\alpha(s-\gamma)^{\beta}} \mathrm{d} s\right)\right\} \\
& +\frac{c_{1}}{T}\left\{I_{\mu} \mathrm{e}^{\alpha(\mu-\gamma)^{\beta}} \int_{\mu}^{\kappa T} \mathrm{e}^{-\alpha(t-\gamma)^{\beta}} \mathrm{d} t-a p^{-b} \omega \eta \mu^{\eta-1}\left(\int_{\mu}^{\kappa T} \mathrm{e}^{-\alpha(t-\gamma)^{\beta}} \mathrm{d} t\right)\left(\int_{0}^{t} \mathrm{e}^{\alpha(s-\gamma)^{\beta}} \mathrm{d} s\right)\right\} \\
& +c_{2} \omega_{a p}{ }^{-b} T^{\eta}\left[\left(\frac{1}{\eta+1}-\kappa^{\eta}\right)+\frac{\eta \kappa^{\eta+1}}{\eta+1}\right]
\end{aligned}
$$

The necessary conditions for minimization of $\phi(T, p)$ are:

$$
\partial \phi(T, p) / \partial T=\partial \phi(T, p) / \partial p=0
$$

The sufficient condition for minimization of $\phi(T, p)$ requires that it must be a convex function for $T>0, p>0$. Now the function $\phi(T, p)$ will be convex if

$$
\left|\begin{array}{ll}
\frac{\partial^{2} \phi(T, p)}{\partial T^{2}} & \frac{\partial^{2} \phi(T, p)}{\partial T \partial p} \\
\frac{\partial^{2} \phi(T, p)}{\partial p \partial T} & \frac{\partial^{2} \phi(T, p)}{\partial p^{2}}
\end{array}\right|>0
$$

Equations (24) can be solved simultaneously by some computer oriented numerical technique such as MathCAD or Maple, to obtain the optimal price $p^{*}$ and optimal cycle time $T^{*}$. We provide a numerical example to illustrate the 
foregoing.

$$
\begin{aligned}
& \frac{\partial \phi(T, p)}{\partial T}=\frac{\partial}{\partial T}\left\{\frac{c_{3} \omega a p^{-b}}{T}\left(\kappa^{n} T^{n}+\eta \mu^{\eta-1}(\kappa T-\mu)+\eta \mu^{\eta-1} \mathrm{e}^{-\alpha(-\gamma)^{\beta}} \int_{0}^{\kappa T} \mathrm{e}^{\alpha(s-\gamma)^{\beta}} \mathrm{d} s\right)\right\} \\
& +\frac{\partial}{\partial T} \frac{c_{1}}{T}\left\{I_{0} \mathrm{e}^{\alpha(-\gamma)^{\beta}} \int_{0}^{\mu} \mathrm{e}^{-\alpha(t-\gamma)^{\beta}} \mathrm{d} t-a p^{-b} \omega \eta \int_{0}^{\mu} \mathrm{e}^{-\alpha(t-\gamma)^{\beta}} \mathrm{d} t \int_{0}^{t} s^{\eta-1} \mathrm{e}^{\alpha(s-\gamma)^{\beta}} \mathrm{d} s\right\} \\
& +\frac{\partial}{\partial T} \frac{c_{1}}{T}\left\{I_{\mu} \mathrm{e}^{\alpha(\mu-\gamma)^{\beta}} \int_{\mu}^{\kappa T} \mathrm{e}^{-\alpha(t-\gamma)^{\beta}} \mathrm{d} t-a p^{-b} \omega \eta \mu^{\eta-1} \int_{\mu}^{\kappa T} \mathrm{e}^{-\alpha(t-\gamma)^{\beta}} \mathrm{d} t \int_{0}^{t} \mathrm{e}^{\alpha(s-\gamma)^{\beta}} \mathrm{d} s\right\} \\
& +\frac{\partial}{\partial T}\left\{c_{2} \omega a p^{-b} T^{\eta}\left[\left(\frac{1}{\eta+1}-\kappa^{\eta}\right)+\frac{\eta \kappa^{\eta+1}}{\eta+1}\right]\right\}
\end{aligned}
$$

Hence,

$$
\begin{aligned}
& \frac{\partial \phi(T, p)}{\partial T}=0 \\
& \Rightarrow-\frac{c_{3} \omega a p^{-b}}{T^{2}}\left(\kappa^{\eta} T^{\eta}+\eta \mu^{\eta-1}(\kappa T-\mu)+\eta \mu^{\eta-1} \mathrm{e}^{-\alpha(-\gamma)^{\beta}} \int_{0}^{\kappa T} \mathrm{e}^{\alpha(s-\gamma)^{\beta}} \mathrm{d} s\right) \\
& +\frac{C_{3} \omega a p^{-b}}{T}\left(\eta \kappa^{\eta} T^{\eta-1}+\kappa \eta \mu^{\eta-1}+\eta \mu^{\eta-1} \mathrm{e}^{-\alpha(-\gamma)^{\beta}} \frac{\partial}{\partial T} \int_{0}^{\kappa T} \mathrm{e}^{\alpha(s-\gamma)^{\beta}} \mathrm{d} s\right) \\
& -\frac{C_{1}}{T^{2}}\left\{I_{0} \mathrm{e}^{\alpha(-\gamma)^{\beta}} \int_{0}^{\mu} \mathrm{e}^{-\alpha(t-\gamma)^{\beta}} \mathrm{d} t-a p^{-b} \omega \eta\left(\int_{0}^{\mu} \mathrm{e}^{-\alpha(t-\gamma)^{\beta}} \mathrm{d} t\right)\left(\int_{0}^{t} s^{\eta-1} \mathrm{e}^{\alpha(s-\gamma)^{\beta}} \mathrm{d} s\right)\right\} \\
& +\frac{c_{1}}{T} \frac{\partial I_{0}}{\partial T} \mathrm{e}^{\alpha(-\gamma)^{\beta}} \int_{0}^{\mu} \mathrm{e}^{-\alpha(t-\gamma)^{\beta}} \mathrm{d} t \\
& -\frac{C_{1}}{T^{2}}\left\{I_{\mu} \mathrm{e}^{\alpha(\mu-\gamma)^{\beta}} \int_{\mu}^{\kappa T} \mathrm{e}^{-\alpha(t-\gamma)^{\beta}} \mathrm{d} t-a p^{-b} \omega \eta \mu^{\eta-1}\left(\int_{\mu}^{\kappa T} \mathrm{e}^{-\alpha(t-\gamma)^{\beta}} \mathrm{d} t\right)\left(\int_{0}^{t} \mathrm{e}^{\alpha(s-\gamma)^{\beta}} \mathrm{d} s\right)\right\} \\
& +\frac{C_{1}}{T}\left\{\frac{\partial I_{\mu}}{\partial T} \mathrm{e}^{\alpha(\mu-\gamma)^{\beta}} \int_{\mu}^{\kappa T} \mathrm{e}^{-\alpha(t-\gamma)^{\beta}} \mathrm{d} t+I_{\mu} \frac{\partial}{\partial T}\left(\int_{\mu}^{\kappa T} \mathrm{e}^{-\alpha(t-\gamma)^{\beta}} \mathrm{d} t\right)\right\} \\
& -\frac{c_{1}}{T}\left\{a p^{-b} \omega \eta \mu^{\eta-1} \frac{\partial}{\partial T}\left(\int_{\mu}^{\kappa T} \mathrm{e}^{-\alpha(t-\gamma)^{\beta}} \mathrm{d} t\right)\left(\int_{0}^{t} \mathrm{e}^{\alpha(s-\gamma)^{\beta}} \mathrm{d} s\right)\right\} \\
& +c_{2} \operatorname{\omega ap}^{-b} \eta T^{\eta-1}\left[\left(\frac{1}{\eta+1}-\kappa^{\eta}\right)+\frac{\eta \kappa^{\eta+1}}{\eta+1}\right]=0 \\
& \frac{\partial \phi(T, p)}{\partial p}=\frac{\partial}{\partial p}\left\{\frac{c_{3} \omega a p^{-b}}{T}\left(\kappa^{n} T^{n}+\eta \mu^{\eta-1}(\kappa T-\mu)+\eta \mu^{\eta-1} \mathrm{e}^{-\alpha(-\gamma)^{\beta}} \int_{0}^{\kappa T} \mathrm{e}^{\alpha(s-\gamma)^{\beta}} \mathrm{d} s\right)\right\} \\
& +\frac{\partial}{\partial p} \frac{c_{1}}{T}\left\{I_{0} \mathrm{e}^{\alpha(-\gamma)^{\beta}} \int_{0}^{\mu} \mathrm{e}^{-\alpha(t-\gamma)^{\beta}} \mathrm{d} t-a p^{-b} \omega \eta\left(\int_{0}^{\mu} \mathrm{e}^{-\alpha(t-\gamma)^{\beta}} \mathrm{d} t\right)\left(\int_{0}^{t} s^{\eta-1} \mathrm{e}^{\alpha(s-\gamma)^{\beta}} \mathrm{d} s\right)\right\} \\
& +\frac{\partial}{\partial p} \frac{c_{1}}{T}\left\{I_{\mu} \mathrm{e}^{\alpha(\mu-\gamma)^{\beta}} \int_{\mu}^{\kappa T} \mathrm{e}^{-\alpha(t-\gamma)^{\beta}} \mathrm{d} t-a p^{-b} \omega \eta \mu^{\eta-1}\left(\int_{\mu}^{\kappa T} \mathrm{e}^{-\alpha(t-\gamma)^{\beta}} \mathrm{d} t\right)\left(\int_{0}^{t} \mathrm{e}^{\alpha(s-\gamma)^{\beta}} \mathrm{d} s\right)\right\} \\
& +\frac{\partial}{\partial p}\left\{c_{2} \omega a p^{-b} T^{\eta}\left[\left(\frac{1}{\eta+1}-\kappa^{\eta}\right)+\frac{\eta \kappa^{\eta+1}}{\eta+1}\right]\right\}
\end{aligned}
$$

Hence,

$$
\frac{\partial \phi(T, p)}{\partial p}=0
$$




$$
\begin{aligned}
\Rightarrow & -\frac{c_{3} \omega a b p^{-b-1}}{T}\left(\kappa^{n} T^{n}+\eta \mu^{\eta-1}(\kappa T-\mu)+\eta \mu^{\eta-1} \mathrm{e}^{-\alpha(-\gamma)^{\beta}} \int_{0}^{\kappa T} \mathrm{e}^{\alpha(s-\gamma)^{\beta}} \mathrm{d} s\right) \\
& +\frac{c_{1}}{T}\left\{\frac{\partial I_{0}}{\partial p} \mathrm{e}^{\alpha(-\gamma)^{\beta}} \int_{0}^{\mu} \mathrm{e}^{-\alpha(t-\gamma)^{\beta}} \mathrm{d} t+a b p^{-b-1} \omega \eta\left(\int_{0}^{\mu} \mathrm{e}^{-\alpha(t-\gamma)^{\beta}} \mathrm{d} t\right)\left(\int_{0}^{t} s^{\eta-1} \mathrm{e}^{\alpha(s-\gamma)^{\beta}} \mathrm{d} s\right)\right\} \\
& +\frac{c_{1}}{T}\left\{\frac{\partial I_{\mu}}{\partial p} \mathrm{e}^{\alpha(\mu-\gamma)^{\beta}} \int_{\mu}^{\kappa T} \mathrm{e}^{-\alpha(t-\gamma)^{\beta}} \mathrm{d} t+a b p^{-b-1} \omega \eta \mu^{\eta-1}\left(\int_{\mu}^{\kappa T} \mathrm{e}^{-\alpha(t-\gamma)^{\beta}} \mathrm{d} t\right)\left(\int_{0}^{t} \mathrm{e}^{\alpha(s-\gamma)^{\beta}} \mathrm{d} s\right)\right\} \\
& -c_{2} \omega a b p^{-b-1} T^{\eta}\left[\left(\frac{1}{\eta+1}-\kappa^{\eta}\right)+\frac{\eta \kappa^{\eta+1}}{\eta+1}\right]=0
\end{aligned}
$$

We recall that;

$$
\begin{gathered}
I_{0}=d(p) \omega \eta \mu^{\eta-1} \mathrm{e}^{-\alpha(-\gamma)^{\beta}} \int_{0}^{t_{1}} \mathrm{e}^{\alpha(s-\gamma)^{\beta}} \mathrm{d} s \\
I(\mu)=d(p) \omega \eta \mu^{\eta-1} \mathrm{e}^{-\alpha(\mu-\gamma)^{\beta}} \int_{\mu}^{t_{1}} \mathrm{e}^{\alpha(s-\gamma)^{\beta}} \mathrm{d} s \\
\frac{\partial I_{0}}{\partial T}=\frac{\partial}{\partial T}\left\{a p^{-b} \omega \eta \mu^{\eta-1} \mathrm{e}^{-\alpha(-\gamma)^{\beta}} \int_{0}^{\kappa T} \mathrm{e}^{\alpha(s-\gamma)^{\beta}} \mathrm{d} s\right\} \\
=a p^{-b} \omega \eta \mu^{\eta-1} \mathrm{e}^{-\alpha(-\gamma)^{\beta}} \frac{\partial}{\partial T} \int_{0}^{\kappa T} \mathrm{e}^{\alpha(s-\gamma)^{\beta}} \mathrm{d} s \\
\frac{\partial I_{0}}{\partial p}=\frac{\partial}{\partial p}\left\{a p^{-b} \omega \eta \mu^{\eta-1} \mathrm{e}^{-\alpha(-\gamma)^{\beta}} \int_{0}^{\kappa T} \mathrm{e}^{\alpha(s-\gamma)^{\beta}} \mathrm{d} s\right\} \\
=-a b p^{-b-1} \omega \eta \mu^{\eta-1} \mathrm{e}^{-\alpha(-\gamma)^{\beta}} \int_{0}^{\kappa T} \mathrm{e}^{\alpha(s-\gamma)^{\beta}} \mathrm{d} s
\end{gathered}
$$

Similarly

$$
\begin{aligned}
\frac{\partial I_{\mu}}{\partial T} & =\frac{\partial}{\partial T}\left\{a p^{-b} \omega \eta \mu^{\eta-1} \mathrm{e}^{-\alpha(\mu-\gamma)^{\beta}} \int_{\mu}^{\kappa T} \mathrm{e}^{\alpha(s-\gamma)^{\beta}} \mathrm{d} s\right\} \\
& =a p^{-b} \omega \eta \mu^{\eta-1} \mathrm{e}^{-\alpha(\mu-\gamma)^{\beta}} \frac{\partial}{\partial T} \int_{\mu}^{\kappa T} \mathrm{e}^{\alpha(s-\gamma)^{\beta}} \mathrm{d} s \\
\frac{\partial I_{\mu}}{\partial p} & =\frac{\partial}{\partial p}\left\{a p^{-b} \omega \eta \mu^{\eta-1} \mathrm{e}^{-\alpha(\mu-\gamma)^{\beta}} \int_{\mu}^{\kappa T} \mathrm{e}^{\alpha(s-\gamma)^{\beta}} \mathrm{d} s\right\} \\
& =-a b p^{-b-1} \omega \eta \mu^{\eta-1} \mathrm{e}^{-\alpha(\mu-\gamma)^{\beta}} \int_{\mu}^{\kappa T} \mathrm{e}^{\alpha(s-\gamma)^{\beta}} \mathrm{d} s
\end{aligned}
$$

The Lebnitz rule for differentiating the integral $I(\alpha)=\int_{a(\alpha)}^{b(\alpha)} f(x, \alpha) \mathrm{d} x$ is given by

$$
\frac{\mathrm{d} I(\alpha)}{\mathrm{d} \alpha}=f(b, \alpha) \frac{\mathrm{d} b}{\mathrm{~d} \alpha}-f(b, \alpha) \frac{\mathrm{d} a}{\mathrm{~d} \alpha}+\int_{a}^{b} \frac{\partial f(x, \alpha)}{\partial \alpha} \mathrm{d} x
$$

Applying this rule to $\frac{\partial}{\partial T} \int_{0}^{\kappa T} I(t, T) \mathrm{d} t$, we get

$$
\begin{gathered}
\frac{\partial}{\partial T} \int_{0}^{\kappa T} I(t, T) \mathrm{d} t=\int_{0}^{\kappa T} \frac{\partial}{\partial T} I(t, T) \mathrm{d} t+\kappa I(\kappa, T) \\
\frac{\partial}{\partial T}\left(\int_{0}^{\kappa T} \mathrm{e}^{\alpha(t-\gamma)^{\beta}} \mathrm{d} t\right)=\int_{0}^{\kappa T} \frac{\partial}{\partial T} \mathrm{e}^{\alpha(t-\gamma)^{\beta}} \mathrm{d} t+\kappa \mathrm{e}^{\alpha(T-\gamma)^{\beta}}=\kappa \mathrm{e}^{\alpha(T-\gamma)^{\beta}}
\end{gathered}
$$

Hence 


$$
\begin{aligned}
\frac{\partial I_{0}}{\partial T} & =a p^{-b} \omega \eta \mu^{\eta-1} \mathrm{e}^{-\alpha(-\gamma)^{\beta}} \frac{\partial}{\partial T} \int_{0}^{\kappa T} \mathrm{e}^{\alpha(s-\gamma)^{\beta}} \mathrm{d} s \\
& =a \kappa p^{-b} \omega \eta \mu^{\eta-1} \mathrm{e}^{-\alpha(-\gamma)^{\beta}+\alpha(T-\gamma)^{\beta}} \\
\frac{\partial I_{\mu}}{\partial T} & =\frac{\partial}{\partial T}\left\{a p^{-b} \omega \eta \mu^{\eta-1} \mathrm{e}^{-\alpha(\mu-\gamma)^{\beta}} \int_{\mu}^{\kappa T} \mathrm{e}^{\alpha(s-\gamma)^{\beta}} \mathrm{d} s\right\} \\
& =a p^{-b} \omega \eta \mu^{\eta-1} \mathrm{e}^{-\alpha(\mu-\gamma)^{\beta}} \frac{\partial}{\partial T} \int_{\mu}^{\kappa T} \mathrm{e}^{\alpha(s-\gamma)^{\beta}} \mathrm{d} s
\end{aligned}
$$

Similarly

$$
\frac{\partial}{\partial T}\left(\int_{\mu}^{\kappa T} \mathrm{e}^{\alpha(t-\gamma)^{\beta}} \mathrm{d} t\right)=\int_{\mu}^{\kappa T} \frac{\partial}{\partial T} \mathrm{e}^{\alpha(t-\gamma)^{\beta}} \mathrm{d} t+\kappa \mathrm{e}^{\alpha(T-\gamma)^{\beta}}=\kappa \mathrm{e}^{\alpha(T-\gamma)^{\beta}}
$$

Hence

$$
\begin{aligned}
\frac{\partial I_{\mu}}{\partial T} & =a p^{-b} \omega \eta \mu^{\eta-1} \mathrm{e}^{-\alpha(\mu-\gamma)^{\beta}} \frac{\partial}{\partial T} \int_{\mu}^{\kappa T} \mathrm{e}^{\alpha(s-\gamma)^{\beta}} \mathrm{d} s \\
& =a \kappa p^{-b} \omega \eta \mu^{\eta-1} \mathrm{e}^{-\alpha(\mu-\gamma)^{\beta}+\alpha(T-\gamma)^{\beta}}
\end{aligned}
$$

\subsection{Solution Algorithm for the Optimal Inventory Policy}

We give the following steps for computing the optimal ordering quantity, optimal cycle time, price and the optimal total cost for the model:

Step 1: Solve Equations (27) and (29) simultaneously to get the optimal values $T^{*}$ and $p^{*}$ for $T$ and $p$ respectively.

Step 2: If at $T^{*}$ and $p^{*}$ the sufficiency condition is satisfied, then go to step 3 else stop and declare the solution infeasible.

Step 3: Substitute $T^{*}$ into $t_{1}=\kappa T$ to obtain $t_{1}^{*}$.

Step 4: Determine the optimal EOQ $I_{0}^{*}$ by substituting the values of $T^{*}$ and $p^{*}$ into eqn. (15).

Step 5: Substitute the values of $I_{0}^{*}, T^{*}$ and $p^{*}$ into Equation (22) to get the optimal total average cost $\phi(T, p)$.

\subsection{Numerical Analysis and Results}

In this section we employ MathCAD 14 computational software [21] to obtain numerical solutions to the highly nonlinear system of Equations (27) and (29). The CAD stands for Computer Aided Design. This will provide us with the optimal solutions for the average cost function for some specified data.

$$
\begin{aligned}
& c_{1}:=2.5 \quad c_{2}:=5 \quad c_{3}:=500 \quad \alpha:=0.01 \quad \beta:=8 \quad \gamma:=0.1 \quad \kappa:=0.75 \\
& a:=15 \cdot 10^{7} \quad b:=3.62 \quad \eta:=2 \quad \omega:=0.1 \quad t:=0.2 \quad \mu:=0.1
\end{aligned}
$$

The format for the MathCAD 14 solve block follows:

- Initial values for the unknown variables $(p, T)$.

- Given.

- Equation(1).

- Equation (2).

- Find $(p, T)$. 


\subsection{MathCAD Solve Block Solution}

$$
\begin{gathered}
c_{1}:=2.5 \quad c_{2}:=5 \quad c_{3}:=500 \quad \alpha:=0.01 \quad \beta:=10 \quad \gamma:=0.1 \quad \kappa:=0.2 \\
a:=15 \cdot 10^{7} \quad b:=3.62 \quad \eta:=2 \quad \omega:=0.1 \quad t:=0.2 \quad \mu:=0.1 \\
p:=70 \quad T:=2 \quad \text { Initial values of the variables }
\end{gathered}
$$

\section{Given}

$$
\begin{aligned}
& -\frac{c_{3} \omega a p^{-b}}{T^{2}}\left(\kappa^{\eta} T^{\eta}+\eta \mu^{\eta-1}(\kappa T-\mu)+\eta \mu^{\eta-1} \mathrm{e}^{-\alpha(-\gamma)^{\beta}} \int_{0}^{\kappa T} \mathrm{e}^{\alpha(s-\gamma)^{\beta}} \mathrm{d} s\right) \\
& +\frac{C_{3} \omega a p^{-b}}{T}\left(\eta \kappa^{\eta} T^{\eta-1}+\kappa \eta \mu^{\eta-1}+\kappa \eta \mu^{\eta-1} \mathrm{e}^{-\alpha(-\gamma)^{\beta}+\alpha(T-\gamma)^{\beta}}\right) \\
& -\frac{c_{1}}{T^{2}}\left\{a p^{-b} \omega \eta \mu^{\eta-1}\left(\int_{0}^{\kappa T} \mathrm{e}^{\alpha(s-\gamma)^{\beta}} \mathrm{d} s\right)\left(\int_{0}^{\mu} \mathrm{e}^{-\alpha(t-\gamma)^{\beta}} \mathrm{d} t\right)\right. \\
& \left.-a p^{-b} \omega \eta\left(\int_{0}^{\mu} \mathrm{e}^{-\alpha(t-\gamma)^{\beta}} \mathrm{d} t\right)\left(\int_{0}^{t} s^{\eta-1} \mathrm{e}^{\alpha(s-\gamma)^{\beta}} \mathrm{d} s\right)\right\} \\
& +\frac{C_{1}}{T} \kappa a p^{-b} \omega \eta \mu^{\eta-1} \mathrm{e}^{\alpha(T-\gamma)^{\beta}} \int_{0}^{\mu} \mathrm{e}^{-\alpha(t-\gamma)^{\beta}} \mathrm{d} t-\frac{C_{1}}{T^{2}}\left\{a p^{-b} \omega \eta \mu^{\eta-1}\left(\int_{\mu}^{\kappa T} \mathrm{e}^{\alpha(t-\gamma)^{\beta}} \mathrm{d} t\right)^{2}\right. \\
& \left.-a p^{-b} \omega \eta \mu^{\eta-1}\left(\int_{\mu}^{\kappa T} \mathrm{e}^{\alpha(t-\gamma)^{\beta}} \mathrm{d} t\right)\left(\int_{0}^{t} \mathrm{e}^{\alpha(s-\gamma)^{\beta}} \mathrm{d} s\right)\right\} \\
& +\frac{C_{1}}{T}\left\{a^{2} \kappa^{2} p^{-2 b} \omega^{2} \eta^{2} \mu^{2(\eta-1)} \mathrm{e}^{-\alpha(\mu-\gamma)^{\beta}+2 \alpha(T-\gamma)^{\beta}}\left(\int_{\mu}^{\kappa T} \mathrm{e}^{\alpha(t-\gamma)^{\beta}} \mathrm{d} t\right)^{2}\right\} \\
& -\frac{C_{1}}{T}\left\{a p^{-b} \kappa \omega \eta \mu^{\eta-1} \mathrm{e}^{\alpha(T-\gamma)^{\beta}} \int_{0}^{t} \mathrm{e}^{\alpha(s-\gamma)^{\beta}} \mathrm{d} s\right\} \\
& +c_{2} \omega a p^{-b} \eta T^{\eta-1}\left[\left(\frac{1}{\eta+1}-\kappa^{\eta}\right)+\frac{\eta \kappa^{\eta+1}}{\eta+1}\right]=0 \\
& -\frac{c_{3} \omega a b p^{-b-1}}{T}\left(\kappa^{\eta} T^{\eta}+\eta \mu^{\eta-1}(\kappa T-\mu)+\eta \mu^{\eta-1} \mathrm{e}^{-\alpha(-\gamma)^{\beta}} \int_{0}^{\kappa T} \mathrm{e}^{\alpha(s-\gamma)^{\beta}} \mathrm{d} s\right) \\
& +\frac{c_{1}}{T}\left\{-a b p^{-b-1} \omega \eta \mu^{\eta-1}\left(\int_{0}^{\kappa T} \mathrm{e}^{\alpha(s-\gamma)^{\beta}} \mathrm{d} s\right)\left(\int_{0}^{\mu} \mathrm{e}^{-\alpha(t-\gamma)^{\beta}} \mathrm{d} t\right)\right. \\
& \left.+a b p^{-b-1} \omega \eta\left(\int_{0}^{\mu} \mathrm{e}^{-\alpha(t-\gamma)^{\beta}} \mathrm{d} t\right)\left(\int_{0}^{t} s^{\eta-1} \mathrm{e}^{\alpha(s-\gamma)^{\beta}} \mathrm{d} s\right)\right\} \\
& +\frac{C_{1}}{T}\left\{-a b p^{-b-1} \omega \eta \mu^{\eta-1}\left(\int_{\mu}^{\kappa T} \mathrm{e}^{\alpha(s-\gamma)^{\beta}} \mathrm{d} s\right)^{2}\right. \\
& \left.+a b p^{-b-1} \omega \eta \mu^{\eta-1}\left(\int_{\mu}^{\kappa T} \mathrm{e}^{\alpha(t-\gamma)^{\beta}} \mathrm{d} t\right)\left(\int_{0}^{t} \mathrm{e}^{\alpha(s-\gamma)^{\beta}} \mathrm{d} s\right)\right\} \\
& -c_{2} \omega a b p^{-b-1} T^{\eta}\left[\left(\frac{1}{\eta+1}-\kappa^{\eta}\right)+\frac{\eta \kappa^{\eta+1}}{\eta+1}\right]=0 \\
& \text { Find }(p, T)=\left(\begin{array}{c}
2.571 \times 10^{4} \\
1.462
\end{array}\right)
\end{aligned}
$$

Optimal cycle time $T^{*}=1.462$ unit, optimal price $p^{*}=25710$ unit, optimal total average cost $\phi^{*}(T, p)=0.00377$ unit. It is numerically verified that this solution satisfies the convexity condition for $\phi(T, p)$.

In summary, for the mathematical model of an inventory system with time dependent three-parameter Weibull deterioration and price-dependent demand in the form of a non-linear function of time, we obtained the following results: 
The optimum cycle time $T^{*}=1.462$ days.

The optimal price $p^{*}=25710$ units.

The optimum stock-period $t_{1}=\kappa T^{*}=0.2 \times 1.462=0.292$ days.

The optimum total average cost $\phi^{*}(T, p)=0.00377$ per day.

The optimum initial inventory level

$I_{0}^{*}=d(p) \omega \eta \mu^{\eta-1} \mathrm{e}^{-\alpha(-\gamma)^{\beta}} \int_{0}^{t_{1}} \mathrm{e}^{\alpha(s-\gamma)^{\beta}} \mathrm{d} s=0.038$ units.

The optimum number of order, $N^{*}=1 / I_{0}^{*}=2.18 \times 10^{13}$ unit-order per day.

\subsection{Sensitivity Analysis}

Sensitivity analysis depicts the extent to which the optimal solution of the model is affected by slight changes or errors in its input parameter values. In the forgoing inventory model, we study the sensitivity of the optimal cycle length $T^{*}$, the price $p^{*}$ and cost $\phi^{*}(T, p)$ with respect to the changes in the values of the parameters $a, b, \alpha, \beta, c_{1}, c_{2}$ and $c_{3}$. The results are shown in Table 1 and Table 2 on the other hand depicts the percentage change in the parameter values from

Table 1 . Sensitivity analysis table.

\begin{tabular}{|c|c|c|c|c|}
\hline Parameter & $\begin{array}{c}\% \text { change in } \\
\text { parameter values }\end{array}$ & $T^{*}$ & $p^{*}$ & $\phi^{*}(T, p)$ \\
\hline \multirow{4}{*}{$a$} & -50 & No convergence & No convergence & - \\
\hline & -20 & No convergence & No convergence & - \\
\hline & +20 & 1.609 & $2.418 \times 10^{4}$ & $1.214 \times 10^{-6}$ \\
\hline & +50 & 1.612 & $2.263 \times 10^{4}$ & $1.546 \times 10^{-6}$ \\
\hline \multirow{4}{*}{$b$} & -50 & No convergence & No convergence & - \\
\hline & -20 & No convergence & No convergence & - \\
\hline & +20 & 1.611 & $2.055 \times 10^{4}$ & $2.190 \times 10^{-6}$ \\
\hline & +50 & 1.620 & $1.793 \times 10^{4}$ & $3.604 \times 10^{-6}$ \\
\hline \multirow{4}{*}{$\alpha$} & -50 & No convergence & No convergence & - \\
\hline & -20 & No convergence & No convergence & - \\
\hline & +20 & 1.548 & $3.763 \times 10^{4}$ & $2.763 \times 10^{-7}$ \\
\hline & +50 & -1.095 & $5.469 \times 10^{4}$ & - \\
\hline \multirow{4}{*}{$\beta$} & -50 & No convergence & No convergence & - \\
\hline & -20 & No convergence & No convergence & - \\
\hline & +20 & 1.744 & $4.414 \times 10^{8}$ & 0.000 \\
\hline & +50 & No convergence & No convergence & - \\
\hline \multirow{4}{*}{$c_{1}$} & -50 & No convergence & No convergence & - \\
\hline & -20 & No convergence & No convergence & - \\
\hline & +20 & -1.192 & $2.393 \times 10^{5}$ & - \\
\hline & +50 & No convergence & No convergence & - \\
\hline \multirow{4}{*}{$c_{2}$} & -50 & No convergence & No convergence & - \\
\hline & -20 & 1.594 & $5.395 \times 10^{4}$ & $6.599 \times 10^{-8}$ \\
\hline & +20 & 1.553 & $3.134 \times 10^{4}$ & $4.622 \times 10^{-7}$ \\
\hline & +50 & 1.41 & $3.189 \times 10^{4}$ & $4.034 \times 10^{-7}$ \\
\hline \multirow{4}{*}{$c_{3}$} & -50 & 1.612 & $6.933 \times 10^{4}$ & $1.346 \times 10^{-8}$ \\
\hline & -20 & 1.6 & $3.121 \times 10^{4}$ & $3.844 \times 10^{-7}$ \\
\hline & +20 & 1.494 & $2.616 \times 10^{4}$ & $1.035 \times 10^{-6}$ \\
\hline & +50 & 1.495 & $2.512 \times 10^{4}$ & $1.499 \times 10^{-6}$ \\
\hline
\end{tabular}


Table 2. Table of change in parameter values.

\begin{tabular}{|c|c|c|c|c|}
\hline \multirow{2}{*}{ Initial values } & \multicolumn{4}{|c|}{$\%$ change in parameter values } \\
\hline & $-50 \%$ & $-20 \%$ & $20 \%$ & $50 \%$ \\
\hline$a$ & $0.5 a$ & $0.8 a$ & $1.2 a$ & $1.5 a$ \\
\hline $15 \times 10^{7}$ & $7.5 \times 10^{7}$ & $1.20 \times 10^{8}$ & $1.80 \times 10^{8}$ & $2.25 \times 10^{8}$ \\
\hline$b$ & $0.5 b$ & $0.8 b$ & $1.2 b$ & $1.5 b$ \\
\hline 3.62 & 1.81 & 2.896 & 4.344 & 5.43 \\
\hline$\alpha$ & $0.5 \alpha$ & $0.8 \alpha$ & $1.2 \alpha$ & $1.5 \alpha$ \\
\hline 0.01 & 0.005 & 0.0025 & 0.012 & 0.015 \\
\hline$\beta$ & $0.5 \beta$ & $0.8 \beta$ & $1.2 \beta$ & $1.5 \beta$ \\
\hline 10 & 5 & 8 & 12 & 15 \\
\hline$c_{1}$ & $0.5 c_{1}$ & $0.8 c_{1}$ & $1.2 \mathrm{c}_{1}$ & $1.5 c_{1}$ \\
\hline 2.5 & 1.25 & 2 & 3 & 3.75 \\
\hline$c_{2}$ & $0.5 c_{2}$ & $0.8 \mathrm{c}_{2}$ & $1.2 \mathrm{c}_{2}$ & $1.5 c_{2}$ \\
\hline 5 & 2.5 & 4 & 6 & 7.5 \\
\hline$c_{3}$ & $0.5 c_{3}$ & $0.8 \mathrm{c}_{3}$ & $1.2 \mathrm{c}_{3}$ & $1.5 c_{3}$ \\
\hline 500 & 250 & 400 & 600 & 750 \\
\hline
\end{tabular}

their initial. The sensitivity analysis is performed by changing each of the parameters by $-50 \%,-20 \%,+20 \%$ and $+50 \%$ taking one parameter at a time and keeping the remaining parameters unchanged. We observe the following:

1) The model has no convergent solution for the $-50 \%$ and $-20 \%$ value of the parameter $a$. For the other percentage values, however, the solution is stable.

2) The model has no convergent solution for the $-50 \%$ and $-20 \%$ value of the parameter $b$. For the other percentage values, the fluctuation in the solution values is negligible.

3) The model has no convergent solution for the $-50 \%$ and $-20 \%$ value of the parameter $\alpha$. For the other percentage values, however, the solution is unstable. Here we obtain a negative value of the optimal cycle length $T^{*}$, which is unrealistic and hence there is no admissible value for the optimal cost $\phi^{*}(T, p)$. Hence the parameter $\alpha$ does not contribute to the sensitivity of the model.

4) The model has no convergent solution for the $-50 \%,-20 \%$ and $+50 \%$ value of the parameter $\beta$.

5) For the inventory holding cost $\left(c_{1}\right)$ per unit per unit time, the model has no convergent solution for the $-50 \%,-20 \%$ and $+50 \%$. Furthermore, we obtain a negative value of the optimal cycle length $T^{*}$ and hence the holding cost does not contribute to the sensitivity of the model.

6) For the shortage cost $\left(c_{2}\right)$ per unit per unit time, the model has no convergent solution for the $-50 \%$ only. For the other percentage values, however, the solution is stable. 
7) For the deteriorated cost $\left(c_{3}\right)$ per unit, we have convergent solutions for all percentage values of this parameter. Furthermore, these solutions are stable.

\section{Conclusion}

In this work we developed an inventory model for a three-parameter Weibull deteriorating items with price-dependent demand rate specified by a nonlinear function of time, and is such that when the price of the commodity increases, demand decreases and when price of a commodity decreases, demand increases. This particular configuration has not been discussed in the literature. Our major contribution is the derivation of the optimal inventory policy for the proposed model and this also involves the necessary and sufficient conditions for the optimal policy. The objective of the model is to minimize the total inventory costs. From the numerical example presented to illustrate the solution procedure of the model, we obtain satisfactory results. We then proceeded $\mathrm{n}$ to perform sensitivity analysis of our model. The sensitivity analysis illustrates the extent to which the optimal solution of the model is affected by slight changes or errors in its input parameter values. It is important to state that the numerical procedure for this problem relied heavily on the power of MathCAD14, which was used to solve a highly nonlinear system of equations in two unknowns, and involving a definite integral. The advantage of this numerical software is that the equations are composed as they appear in the text and need not be recast in a special format for computation.

\section{Conflicts of Interest}

The authors declare no conflicts of interest regarding the publication of this paper.

\section{References}

[1] Harris, F. (1915) Operations and Costs (Factory Management Series). A.W. Shaw Co., Chicago, 18-52.

[2] Chakrabarti, T., Giri, B.C. and Chaudhuri, K.S. (1998) An EOQ Model for Items with Weibull Distribution Deterioration, Shortages and Trended Demand. An Extension of Philip's Model. Computer and Operations Research, 25, 649-657. https://doi.org/10.1016/S0305-0548(97)00081-6

[3] Covert, R.P. and Philip, G.C. (1973) An EOQ Model for Items with Weibull Distribution Deterioration. AIIE Transactions, 5, 323-326. https://doi.org/10.1080/05695557308974918

[4] Datta, T.K. and Pal, A.K. (1988) Order Level Inventory System with Power Demand Pattern for Items with Variable Rate of Deterioration. Indian Journal of Pure and Applied Mathematics, 19, 1043-1053.

[5] Jalan, A.K., Giri, R.R. and Chaudhuri, K.S. (1996) EOQ Model for Items with Weibull Distribution Deterioration, Shortages and Trended Demand. International Journal of System Science, 27, 851-855. https://doi.org/10.1080/00207729608929285

[6] Dixit, V. and Shah, N.H. (2006) An Order Level Inventory Model with Decreasing Demand and Time Dependent Deterioration. The International Journal of Man- 
agement Science, 22, 70-78.

[7] Giri, S.C. and Goyal, S.K. (2001) Recent Trends in Modelling of Deteriorating Inventory. European Journal of Operational Research, 134, 1-16. https://doi.org/10.1016/S0377-2217(00)00248-4

[8] Mahata, G.C. and Goswami, A. (2009) A Fuzzy Replenishment Policy for Deteriorating Items with Ramp Type Demand Rate under Inflation. International Journal of Operational Research, 5, 328-348. https://doi.org/10.1504/IJOR.2009.025200

[9] Nwoba, P.O., Chukwu, W.I.E. and Maliki, O.S. (2019) Analysis of an Inventory System for Items with Stochastic Demand and Time Dependent Three-Parameter Weibull Deterioration Function. A.M., 10, 728-742.

https://doi.org/10.4236/am.2019.109052

[10] Naddor, E. (1966) Inventory Systems. John Wiley \& Sons, New York.

[11] Ritchie, E. (1985) Stock Replenishment Quantities for Unbounded Linear Increasing Demand: An Interest Consequence of the Optimal Policy. Journal of the Operational Research Society, 36, 737-739. https://doi.org/10.1057/jors.1985.131

[12] Garg, G., et al. (2012) An EPQ Model with Price Discounting for Non-Instantaneous Deteriorating Item with Ramp-Type Production and Demand Rates. International Journal of Computer \& Mathematical Sciences, 7, 513-554.

[13] Dave, U. (1986) An Order Level Inventory for Items with Variable Instantaneous Demand and Discrete Opportunities for Replenishment. Opsearch, 23, 244-249.

[14] Li, R., Lan, H. and Mawhhinney, J.R. (2010) A Review on Deteriorating Inventory Study. Journal of Service Science and Management, 3, 117-129. https://doi.org/10.4236/jssm.2010.31015

[15] Khanra, S., Ghosh, S.K. and Chaudhuri, K.S. (2011) An EOQ Model for a Deteriorating Item with Time-Dependent Quadratic Demand under Permissible Delay in Payment. Applied Mathematics and Computation, 218, 1-9. https://doi.org/10.1016/j.amc.2011.04.062

[16] Maiti, A.K., Maiti, M.K. and Maiti, M. (2009) Inventory Model with Stochastic Lead-Time and Price Dependent Demand Incorporating Advance Payment. Applied Mathematical Modelling, 33, 2433-2443. https://doi.org/10.1016/j.apm.2008.07.024

[17] Chung, C.S., Flynn, J. and Zhu, J. (2009) The Newsvendor Problem with an In-Season Price Adjustment. European Journal of Operational Research, 198, 148-156. https://doi.org/10.1016/j.ejor.2007.10.067

[18] Rinne, H. (2009) The Weibull Distribution: A Handbook. Chapman \& Hall/CRC, London. https://doi.org/10.1201/9781420087444

[19] Ghosh, S.K. and Chaudhuri, K.S. (2004) An Order-Level Inventory Model for a Deteriorating Item with Weibull Distribution Deterioration, Time-Quadratic Demand and Shortages. Advanced Modeling and Optimization, 6, 21-35.

[20] Saha, S. and Chakrabarti, T. (2019) An EOQ Inventory Model for Time Dependent Deteriorating Items under Price Dependent Ramp Type Demand with Shortages. International Journal of Advanced Research in Science, Engineering and Technology, 6, 10476.

[21] Mathcad Version 14 (2007) PTC (Parametric Technology Corporation) Software Products. http://ptc.com 